\title{
THE HUBBLE SPACE TELESCOPE KEY PROJECT TO MEASURE THE HUBBLE CONSTANT
}

\author{
WENDY L. FREEDMAN \\ Carnegie Observatories \\ 813 Santa Barbara St., Pasadena CA 91106 \\ JEREMY R. MOULD \\ Mount Stromlo and Siding Spring Observatories \\ Australian National University, Weston Creek, Australia \\ ROBERT C. KENNICUTT JR. \\ Steward Observatory \\ University of Arizona, Tucson AZ 85721 \\ AND \\ BARRY F. MADORE \\ IPAC/Caltech \\ 100-25, Pasadena, CA 91125
}

\section{Introduction}

A Joint Discussion on the extragalactic distance scale and the Hubble constant took place fifteen years ago, at the 1982 XVIIIth General Assembly of the IAU, held in Patras, Greece. At that time, the newest applications of infrared photometers to Tully-Fisher measurements (Aaronson 1983) and Cepheid distances (Madore 1983) were reported. CCDs were just coming into use and had not yet been applied to extragalactic distance determinations; all of the extragalactic Cepheid distances were based on photographic Argelander (eye-estimated) photometry (Tammann and Sandage 1983 and references therein). No Cepheid distances to type Ia supernova-host galaxies were available.

What is the situation in 1997 at the time of IAU Symposium 183 in Kyoto? Since Patras, we have seen a steady increase in the precision with which extragalactic distances can be measured. With the widespread availability of linear array detectors, more accurate distances to both the primary and secondary distances are being obtained (e.g., Jacoby et al. 1992; Freedman 
\& Madore 1996; Donahue \& Livio 1997). In parallel to the advances in detector technology has been the development of several new and independent techniques for measuring distances. For the first time in the history of this difficult field, relative distances to galaxies are being compared on a case-by-case basis, and their quantitative agreement is being established. Briefly, we review here progress on the Hubble Space Telescope Key Project to measure $\mathrm{H}_{0}$.

\section{The $\mathbf{H}_{0}$ Key Project}

Converging on an accurate value of the Hubble constant has been a slow and incremental process. The difficulties have stemmed largely from the effects of systematic errors in the extragalactic distance scale. For this reason, the Key Project has been designed to incorporate many independent cross-checks of both the primary and secondary distance scales. Rather than concentrate on one particular method, the goal of the Key Project is to undertake a comparison and a calibration of several different methods so that cross-checks on both the absolute zero point as well as relative distances, and therefore on $\mathrm{H}_{0}$, can be obtained.

Ultimately, the aim of the Key Project is to derive a value for the expansion rate of the Universe, the Hubble constant, to an accuracy of $10 \%$ (Freedman et al. 1994a; Kennicutt, Freedman \& Mould 1995; Mould et al. 1995; Madore et al. 1998). It has been designed with three primary goals: (1) to discover Cepheids, and thereby measure accurate distances to spiral galaxies located in the field and in small groups that are suitable for the calibration of several independent secondary methods, (2) to make direct Cepheid measurements of distances to three spiral galaxies in each of the Virgo and Fornax clusters, and (3) to provide a check on potential systematic errors both in the Cepheid distance scale and the secondary methods. We briefly review the progress in each of these areas.

\section{Measurement of Cepheid Distances / Calibration of Secondary Methods}

To date the $\mathrm{H}_{0}$ Key Project results have been published for M81 (Freedman et al. 1994b), M100 (Ferrarese et al. 1996; Freedman et al. 1994a), M101 (Kelson et al. 1996, 1997), NGC 925 (Silbermann et al. 1996), and NGC 3351 (Graham et al. 1997). Recently, we have also determined distances to NGC 3621 (Rawson et al. 1998), NGC 2090 (Phelps et al. 1998), NGC 7331 (Hughes et al. 1998), NGC 4414 (Turner et al. 1998), and NGC 1365 (Silbermann et al. 1998; Madore et al. 1998). Significant progress has also been made in the HST supernova calibration project; Cepheids have been located and studied in IC 4182 (Saha et al. 1994), NGC 5253 
(Saha et al. 1995) and NGC 4536 (Saha et al. 1996). Results have also been published for NGC 4639 and NGC 4496A (Sandage et al. 1996), and Cepheids have been detected in the Leo I galaxy NGC 3368 (M96) by Tanvir et al. (1995).

To minimize the risk of systematic errors in the data reduction phase, all of the reductions within the Key Project effort are undertaken by two independent groups, using two different software packages: DoPHOT and ALLFRAME (Schechter et al. 1993; Saha et al. 1994; Stetson 1994). In addition, we are currently performing a series of artificial star tests to better quantify the effects of crowding, and to understand the limits in each of these packages (Ferrarese et al., 1998 in preparation). Because our requirement for an accurate, absolute calibration is critical, we are also undertaking an extensive, independent calibration of the WFPC2 zero point (Stetson et $a l$. , in preparation), complementary to the efforts of the WFPC2 instrument team and the Space Telescope Science Institute.

Determination of $\mathrm{H}_{0}$ to an accuracy of $10 \%$ requires that measurements be acquired at great enough distances and in a variety of directions so that the average contribution from peculiar motions of galaxies is significantly less than $10 \%$ of the overall expansion velocity. The current limit for detection of Cepheids with HST is a distance of about 25-30 Mpc (Newman et al. 1998; Saha et al. 1996), where peculiar motions can still contribute $10-20 \%$ of the observed velocity. Hence, the main thrust of the Key Project is the calibration of secondary distance indicators which then operate out to distances significantly greater than can be measured with Cepheids alone. The calibrating galaxies in the sample all have velocities of less than $\sim 1,500 \mathrm{~km} / \mathrm{sec}$. Even with the relative proximity of these galaxies, discovering Cepheids remains a challenging, time-consuming task using HST; the integration times for the more distant galaxies in the sample can each amount to over 30 orbits of HST time.

With the database of Cepheid distances being assembled as part of the $\mathrm{H}_{0}$ Key Project, a number of secondary indicators can be directly calibrated and tested. Several of these methods can be applied to velocity-distances of $10,000 \mathrm{~km} / \mathrm{sec}$ or greater. These include type Ia supernovae, type II supernovae, the Tully-Fisher relation, and the $\mathrm{D}_{n}-\sigma$ relation. Applicable at intermediate distances is the surface-brightness fluctuation method (e.g., Tonry et al. 1997). Although the planetary nebula luminosity function method (e.g., Feldmeirer, Ciardullo \& Jacoby 1997) only extends over the same range as the Cepheids (out to about $20 \mathrm{Mpc}$ ), it offers a valuable comparison and test of methods that operate locally (Cepheids, RR Lyrae stars, tip of the red giant branch (TRGB)) and those that operate at intermediate and greater distances (e.g., surface-brightness fluctuations and the Tully-Fisher relation). The database of Cepheid distances will also provide 
a means for evaluating as yet less well-tested methods; for instance, the globular cluster luminosity function (for a recent application see Baum et al. 1997), red supergiants, and HII region diameters.

In the limited space available here, we confine our remarks to the calibration of the Tully-Fisher relation and type Ia supernovae.

\subsection{CALIBRATION OF THE TULLY-FISHER RELATION}

One of the key elements of the HST $\mathrm{H}_{0}$ Key Project is the Cepheid calibration of the relation between the luminosity and rotational velocity of spiral galaxies, the Tully-Fisher (TF) relation. On the basis of their relatively high inclinations, line widths, and late morphological types, fourteen of our target galaxies were chosen to be useful as Tully-Fisher calibrators. These include NGC 3031, 925, 3351, 2090, 7331, 3621, 2541, 3198, 3319, 4725, 4535, 4548, 1365, and 1425. Along with NGC 3368 (Tanvir et al. 1995), NGC 4536 and 4639 observed by Sandage and collaborators, and the TF galaxies that have had Cepheid distances determined from the ground, NGC 598, 224, 2403 and 300 (Freedman 1990; Madore \& Freedman 1991), this yields a total of 21 individual TF calibrators. The new HST distances increase by a factor of 4 the numbers of TF calibrators previously available from ground-based Cepheid searches.

The status of the $\mathrm{H}_{0}$ Key Project Tully-Fisher calibration has been reviewed recently by Mould et al. (1997). This preliminary calibration yields a value of $\mathrm{H}_{0}=73 \pm 10 \mathrm{~km} / \mathrm{sec} / \mathrm{Mpc}$. This value is in very good agreement with a recent analysis of 24 clusters with Tully-Fisher measurements by Giovanelli et al. (1997). Based on a similar set of Cepheid distances, these authors find $\mathrm{H}_{0}=69 \pm 5 \mathrm{~km} / \mathrm{sec} / \mathrm{Mpc}$.

For illustration, we show in Figure 1 an $\mathrm{H}$-band Tully-Fisher relation for the calibrating galaxies with measured Cepheid distances available to date. The H-band photometry and line widths are from Aaronson et al. (1982); these data (including lower line-width systems) are presented by Mould et al. (1997). Distances to the galaxies are from Freedman (1990), Freedman et al. (1994), Tanvir et al. (1995), Silbermann et al. (1996), Graham et al. (1997), Rawson et al. (1997), and Hughes et al. (1998). There are 11 galaxies plotted. As part of the Key Project, we have obtained and are currently analyzing new UBVRIJHK images of all of the calibrating galaxies for calibration purposes.

\subsection{CALIBRATION OF TYPE IA SUPERNOVAE}

One of the most promising methods for measuring relative distances to distant galaxies is based on the measurement of type Ia supernovae luminosities. Cepheid calibrators have recently become available for this method as 


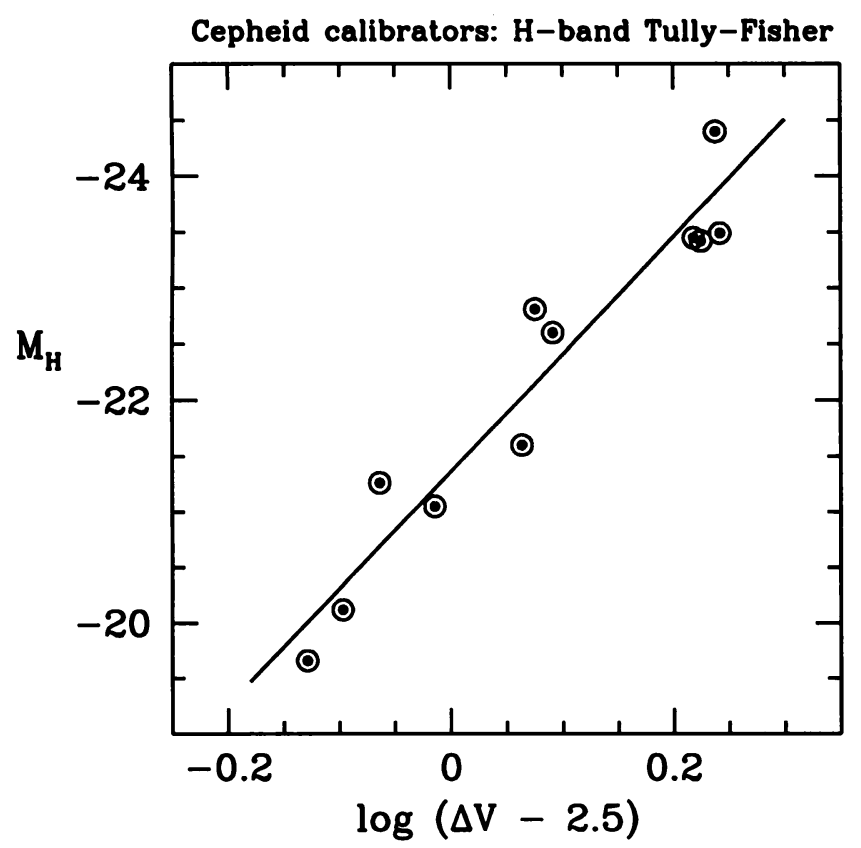

Figure 1. H-band Tully-Fisher relation for galaxies with distances determined from Cepheid variables.

a result of the availability of HST (e.g., Sandage et al. 1995 and references therein). Several independent studies now suggest that type Ia supernovae are not the simple standard candles that they were earlier suggested to be, but they appear to obey a fairly well-defined relation between the absolute magnitude at maximum light and the shape or decline rate of the supernova light curve (Phillips 1993; Hamuy et al. 1995, 1996; Reiss, Press \& Kirshner 1995).

As part of the Key Project target sample, we have observed NGC 4414 (Turner et al. 1998, in preparation), an inclined spiral galaxy useful as both a Tully-Fisher calibrator as well as a type Ia supernova calibrator. It was host to supernova 1974G; unfortunately, however, the light curve of this supernova is of only moderate quality. The Fornax cluster elliptical galaxies NGC 1316 and NGC 1380 are each hosts to the well-observed type Ia supernovae $1980 \mathrm{~N}$ and $1992 \mathrm{~A}$, respectively. (The supernova $1981 \mathrm{D}$ was also observed in NGC 1316, but the data are photographic, and hence are not of as high quality as the other two.)

The new Cepheid distance to NGC 1365, and associated estimate of the distance to the Fornax cluster (discussed below) thus allow two additional very high-quality objects to be added to the calibration of type 
Ia supernovae. Including the the Fornax cluster supernovae $(1980 \mathrm{~N}$ and 1992A), in addition to the other Cepheid calibrators of the Sandage et al. program (1937C, 1972E, 1960F, 1981B, 1989B, and 1990N), and applying this calibration to the distant type Ia supernovae of Hamuy (1995) gives $\mathrm{H}_{0}=64-68 \mathrm{~km} / \mathrm{sec} / \mathrm{Mpc}$ (Freedman et al. 1997). The larger value of $\mathrm{H}_{0}$ compared to that of Sandage et al. (1996) $(57 \mathrm{~km} / \mathrm{sec} / \mathrm{Mpc})$ is due to three factors: (1) we have given low weight to historical supernovae observed photographically, (2) we have included a decline-rate absolute-magnitude relation, and (3) we have added 2 new Fornax calibrators. All three factors contribute in roughly comparable proportions. We note that the addition of the Fornax calibrators changes the value of $\mathrm{H}_{0}$ by $+3 \mathrm{~km} / \mathrm{sec} / \mathrm{Mpc}$ or less than $5 \%$; most of the remaining difference reflects the lower weight given to the historical supernovae 1895B, 1937C, and 1961F.

\section{The Virgo/Fornax clusters and the Local Cepheid Calibration}

The current limit for the detection of Cepheids with HST appears to have been reached at a velocity of $\sim 3,000 \mathrm{~km} / \mathrm{sec}$ (Newman et al. 1998). Hence, the existence of large-scale flows still precludes the current measurement of $\mathrm{H}_{0}$ to $\pm 10 \%$ with Cepheids alone. However, direct Cepheid distances to the Virgo and Fornax clusters at $\mathrm{cz} \geq 1,200 \mathrm{~km} / \mathrm{sec}$ can still provide a consistency check at a level of $\pm 20 \%$.

The Virgo cluster is not an ideal cluster for either the determination of $\mathrm{H}_{0}$ or the calibration of secondary methods. As discussed in detail in Freedman et al. (1994a), one of the dominant uncertainties in the determination of $\mathrm{H}_{0}$ based on the Virgo cluster is due to the fact that the distribution of its spiral galaxies is both extended and complex. As such, a single galaxy alone cannot define the mean distance to the Virgo cluster to an accuracy of better than 15-20\% (Freedman et al. 1994a, Mould et al. 1995). Cepheid distances to five spiral galaxies in the Virgo galaxy have now been published, and they are listed in Table 1. Despite all of the complications, it is interesting that the mean Cepheid distance agrees very well with recent independent estimates of the Virgo cluster distance obtained by Jacoby et al. (1997) and Tonry et al. (1997) for elliptical galaxies.

Adopting a recession velocity for the Virgo cluster of $1,404 \pm 80 \mathrm{~km} / \mathrm{sec}$ (Huchra 1988) and a Virgo distance of $17.8 \mathrm{Mpc}$ yields a value of $\mathrm{H}_{0}=$ $79 \pm 6$ (random) \pm 16 (systematic) $\mathrm{km} / \mathrm{sec} / \mathrm{Mpc}$. Alternatively, adopting a recession velocity of $1,179 \pm 17 \mathrm{~km} / \mathrm{sec}$ (Jerjen and Tammann 1993) results in $\mathrm{H}_{0}=66 \pm 14 \mathrm{~km} / \mathrm{sec} / \mathrm{Mpc}$ for the same distance. The dominant sources of uncertainty in this estimate are systematic: (a) reddening corrections, (b) the zero point of the Cepheid PL relation, (c) the position of these galaxies with respect to the center of the Virgo cluster, and (d) the adopted recession 
velocity of the cluster.

In addition to the galaxies listed in Table 1, two additional Virgo cluster galaxies (NGC 4535 and NGC 4548), have been observed with HST and are currently being analyzed as part of the Key Project. However, despite the increase in numbers of Virgo cluster galaxies with Cepheid distances, the clumpiness, large angular extent, and unknown peculiar motion of the Virgo cluster all preclude a determination of the Hubble constant at a level better than $\sim \pm 20 \%$. A more favorable cluster for allowing a consistency check of $\mathrm{H}_{0}$ determinations from secondary distance indicators is the Fornax cluster.

TABLE 1. CEPHEID DISTANCES TO VIRGO CLUSTER GALAXIES

\begin{tabular}{ccc}
\hline Galaxy & Distance Modulus & Distance \\
\hline NGC 4321 & $31.04 \pm 0.21$ & $16.1 \pm 1.5$ \\
NGC 4496A & $31.13 \pm 0.10$ & $16.8 \pm 0.8$ \\
NGC 4571 & $30.87 \pm 0.15$ & $14.9 \pm 1.2$ \\
NGC $4536^{1}$ & $31.10 \pm 0.13$ & $16.6 \pm 1.0$ \\
NGC 4639 & $32.00 \pm 0.23$ & $25.1 \pm 2.5$ \\
& & \\
1 & $31.25 \pm 0.20$ & $17.8 \pm 1.8$ \\
\hline Mean & 4536 corrected for "long" zero point by +0.05 mag
\end{tabular}

Recently we have analyzed a sample of 37 newly-discovered Cepheids in the galaxy NGC 1365 in the Fornax cluster (Silbermann et al. 1998; Madore et al. 1998). These preliminary results have been previously reported in Freedman et al. (1997). Two additional galaxies in the Fornax cluster have now been observed, and are currently being analyzed: NGC $1326 \mathrm{~A}$ and NGC 1425. The Fornax cluster is a particularly important cluster because it is very compact and contains galaxies with a range of morphological types. In contrast to the Virgo cluster, the small angular size of the Fornax cluster makes the determination of its distance much more straightforward. Thus, it can provide an important calibration of several secondary methods. Of particular interest is the fact that the Fornax cluster contains two wellobserved recent type Ia supernovae, which allows for a direct comparison between the type Ia distance scale and other well-studied secondary indi- 
cators with small measured dispersions, such as the Tully-Fisher relation, surface brightness fluctuations, and planetary nebula luminosity function.

Correcting for a derived total line-of-sight reddening of $E(V-I)=$ $0.10 \mathrm{mag}$ (derived from the NGC 1365 Cepheids themselves) gives a true distance modulus of $\mu_{0}=31.3 \pm 0.1 \mathrm{mag}$ (Silbermann et al. 1998). This corresponds to a distance to NGC 1365 of $18.2 \pm 1.0 \mathrm{Mpc}$. This distance agrees well with the distances to the Fornax cluster determined previously by Jacoby et al. (1997) and Tonry et al. (1997).

A determination of the Hubble constant at the distance of the Fornax cluster requires a knowledge of the Local Group infall velocity to the Virgo cluster. Fortunately, however, the derived infall correction to Fornax is quite insensitive to the assumed infall velocity to Virgo: for an infall velocity of $+200 \pm 100 \mathrm{~km} / \mathrm{sec}$ the flow correction for Fornax is only -40 $\pm 20 \mathrm{~km} / \mathrm{sec}$, yielding a cosmological expansion rate of Fornax (determined from the barycentre of the Local Group) of $1,330 \mathrm{~km} / \mathrm{sec}$ (Madore et al. 1998). Using our Cepheid distance of $18.2 \mathrm{Mpc}$ for Fornax gives $H_{0}=73$ \pm 3 (statistical) \pm 18 (systematic) $\mathrm{km} / \mathrm{sec} / \mathrm{Mpc}$.

To conclude this section on local $\mathrm{H}_{0}$ determinations, an average of the six independent determinations based on nearby galaxies and groups, including the Virgo and Fornax clusters, gives $H_{0}=75 \pm 15 \mathrm{~km} / \mathrm{sec}$ (Madore et al. 1998). It should be noted that the determinations of $H_{0}$ in this section make no explicit allowance for the possibility that the inflow-corrected velocities of nearby clusters could be perturbed significantly by other mass concentrations or large-scale flows beyond the Virgo cluster. However, it is interesting to note that these local estimates agree very well with the determinations of $\mathrm{H}_{0}$ at larger distances, where peculiar velocities are a fractionally-smaller uncertainty $(\S 3.1,3.2)$.

\subsection{TESTS OF THE CEPHEID DISTANCE SCALE}

A number of tests on the Cepheid distance scale are currently feasible. Space here precludes a detailed discussion of many of these efforts, but in brief, comparisons of Cepheid distances with other independently-calibrated distance indicators (e.g., RR Lyraes, and tip of the red giant branch (TRGB)) (e.g., see the reviews by Freedman \& Madore 1993; Freedman \& Madore 1996 ; Madore, Freedman \& Sakai 1997) distances agree with the Cepheid distances at a level of $\pm 0.1 \mathrm{mag} r m s$ (Lee et al. 1993; Sakai et al. 1996). The agreement between the absolute calibrations of the RR Lyraes (upon which the TRGB distances are based) and the Cepheids is still a matter of some debate, with an $r m s$ uncertainty of $\pm 0.1 \mathrm{mag}$. This uncertainty lies at the heart of the current uncertainty in the distance modulus to the Large Magellanic Cloud, $\mu=18.5 \pm 0.1 \mathrm{mag}(e . g .$, , see the review by Westerlund 
1997), which currently provides the fiducial period-luminosity relations for extragalactic distances.

Distances to galaxies based on Cepheids can be compared on a relative basis with a number of other indicators including the tip of the red giant branch, planetary nebula luminosity function, surface brightness fluctuations, and types I and II supernovae. The agreement in these cases is also quantitatively very encouraging. For a comparison of recent Cepheid distances obtained as part of the $\mathrm{H}_{0}$ Key Project with those based on these other methods, see Freedman, Madore \& Kennicutt (1997).

\subsection{IS THERE A SIGNIFICANT METALLICITY DEPENDENCE?}

A potentially important systematic effect on the Cepheid distance scale is the metal abundance. To date, there has been no consensus on how significant such an effect might be. Unfortunately, theory cannot currently provide a definitive answer to the issue of how abundance affects the observed luminosities of Cepheids. Recent models by Chiosi, Wood \& Capitanio (1993) suggest that at the V and I wavebands observed with HST, the effect of abundance amounts to approximately $-0.1 \mathrm{mag} / \mathrm{dex}$. Earlier models by Stothers (1988) and Iben and Tuggle (1975) were based on B and $\mathrm{V}$ photometry, and predicted a much larger effect (see Freedman \& Madore 1990 for a summary of these results). According to these models, both the sense and the magnitude of the effects of metallicity are dependent on wavelength: in the blue, higher metallicity Cepheids appear fainter due to line blanketing. The magnitude effect is smaller in the red, and the redistribution of the line-blanketed radiation makes the Cepheids appear brighter. More recent work incorporating new opacities, by Chiosi, Wood \& Capitanio (in preparation), predicts a smaller effect, $0.06 \mathrm{mag} / \mathrm{dex}$, but the sign is now in the opposite sense. Hence, the theoretical predictions are not yet firm and empirical studies are critical for placing limits on the magnitude of any abundance effects.

At present, the observational situation also remains unresolved. The first observational test for an abundance dependence of the Cepheid periodluminosity relation was undertaken by Freedman and Madore (1990). These authors observed samples of Cepheids at three positions in M31 with respect to the radial gradient in metallicity. They concluded that, after correcting for reddening, the difference in true modulus that could be attributed to metallicity was less than $10 \%$ over a range in abundance of a factor of $\sim 3$. These data were subsequently reanalyzed by Gould (1994) who concluded, to the contrary, that over a range in metallicity of $1 \mathrm{dex}$, a 0.56 to $0.88 \mathrm{mag}$ difference would be measurable.

The results of Gould (1994), however, are inconsistent with other limits 
from comparisons of Cepheid distances with completely independent distance methods such as the TRGB method discussed above. For a wide range of both TRGB and Cepheid metallicities, the relative distances between the two methods agree to within the 1- $\sigma$ uncertainties of each of the methods. As stated above, the rms differences amount to less than 0.1 mag.

More recently, Sasselov et al. (1997) and Beaulieu et al. (1997) have analyzed data taken as part of the EROS search for MACHOS in the LMC and SMC. They find a dependence of $-0.44_{-0.2}^{+0.1} \mathrm{mag} / \mathrm{dex}$. This value is similar to the value obtained by Kochanek (1997) based on an analysis of Cepheid data for a number of galaxies obtained from a variety of sources, and with a variety of bandpasses. An analysis of Galactic Cepheids by Sekiguchi \& Fukugita (1997) finds a much steeper dependence, very strongly in conflict with the constraints provided by other distance indicators.

As part of the Key Project, we have undertaken a second empirical test in two fields in the face-on spiral galaxy M101. We find a small dependence on metallicity, again with a large uncertainty: $\Delta \mu_{0} / \Delta[\mathrm{O} / \mathrm{H}]=-0.24$ $\pm 0.16 \mathrm{mag} / \mathrm{dex}$ (Kennicutt et al. 1998). Again, comparison of the Key Project Cepheid distances with other distance indicators provides a strong constraint on the size of a metallicity effect. The metallicity of the galaxies for which Cepheid searches have been undertaken span a range in $[\mathrm{O} / \mathrm{H}]$ abundance of almost an order of magnitude, with a median value of -0.3 dex. The Large Magellanic Cloud, which currently provides the calibrating period-luminosity relation for extragalactic distances, has a very similar abundance of $[\mathrm{O} / \mathrm{H}]=-0.4 \mathrm{dex}$. These results suggest that in individual cases the metallicity effect may amount to $10 \%$, but the overall effect on the calibration of secondary distance indicators will be less than a few percent.

Further progress on constraining the size of a metallicity dependence will come from NICMOS observations of Cepheids with a range of metallicities, currently scheduled for this upcoming HST cycle. At long wavelengths, the reduced sensitivity to both reddening and metallicity will improve the accuracy in the resulting distances by a factor of $>2$.

\subsection{RECENT RESULTS FROM HIPPARCOS}

Feast and Catchpole (1997) have recently published the first results on parallaxes to Galactic Cepheids based on measurements from the Hipparcos satellite. Based on data for the 26 highest signal-to-noise Cepheid parallaxes, they calibrate the zero point of the V-band Galactic Cepheid periodluminosity relation, adopting the slope from prior work on LMC Cepheids. Correcting for $E(B-V)_{L M C}=0.074 \mathrm{mag}$, adding a theoretical metallicity correction of $+0.042 \mathrm{mag}$, and adopting $\langle V\rangle_{o}-\log (P)$ from Caldwell \& Laney (1991), they derive a distance modulus $\left(m-M_{V}\right)_{o}^{L M C}=18.70 \pm$ 
0.10 mag, based on the V-band PL relation.

Madore \& Freedman (1997) have also calibrated the Cepheid periodluminosity relation based on the Hipparcos parallaxes for Galactic Cepheids published by Feast \& Catchpole (1997), but at six wavelengths (BVIJHK). Unfortunately, the current parallax errors for the fundamental pulsators are very large (they range in signal-to-noise $=\pi / \sigma_{\pi}$ from 0.3 to 5.3 , at best) and they preclude an unambiguous interpretation of the observed differences. These differences may arise from a combination of true distance modulus, reddening and/or metallicity effects. Currently extragalactic distances are calibrated relative to those of the Large Magellanic Cloud (LMC). These results suggest a range of LMC moduli between $18.44 \pm 0.35$ and 18.57 $\pm 0.11 \mathrm{mag}$ ( 49 to $52 \mathrm{kpc}$ ). Comparing these calibrations with previously published multiwavelength PL relations from Madore \& Freedman (1991), there is very good agreement at a level of $0.07 \pm 0.14 \mathrm{mag}$, or $4 \pm 7 \%$ in distance. Madore and Freedman adopted 18.50 $\pm 0.10 \mathrm{mag}$ for the distance to the LMC.

Recently, there have been a number of other independent measurements of the distance to the LMC. A new, independent measurement of the RR Lyrae distance to the LMC yields $18.48 \pm 0.19 \mathrm{mag}$ (Alcock et al. (1997). Based on an analysis of the expanding ring of supernova 1987A, Gould \& Uza (1997) derive $\mu_{L M C}<18.37 \pm 0.04 \mathrm{mag}$ for the LMC true distance modulus if the ring is assumed to be circular; they note that if the ring is slightly elliptical $(b / a \sim 0.95)$ this upper limit increases to $<18.44 \pm 0.05 \mathrm{mag}$. A value of $18.56 \pm 0.05 \mathrm{mag}$ has been derived by Panagia et al. (1996) from the same data. For the present time, the $\mathrm{H}_{0}$ Key Project has adopted a true distance modulus of $18.50 \pm 0.10 \mathrm{mag}$ for the LMC. This value is consistent with other estimated distances to the LMC based on a wide variety of methods (e.g., Westerlund 1997).

\section{Summary}

Fifteen years ago at the 1982 General Assembly, there was no reconciling the values of $\mathrm{H}_{0}$ obtained from the infrared Tully-Fisher relation presented by the late Marc Aaronson $\left(\mathrm{H}_{0}=85 \pm 5 \mathrm{~km} / \mathrm{sec} / \mathrm{Mpc}\right.$; Aaronson 1983), the value of $50 \pm 7 \mathrm{~km} / \mathrm{sec} / \mathrm{Mpc}$ presented by Gustav Tammann and Allan Sandage based primarily on type Ia supernovae calibrated by $M$ supergiants via Cepheids, and the value of $\mathrm{H}_{0}=95 \pm 10 \mathrm{~km} / \mathrm{sec} / \mathrm{Mpc}$ derived by the late Gerard de Vaucouleurs, based on a number of different methods. At the IAU Symposium 183 in 1997, we appear to be seeing some convergence in values of $\mathrm{H}_{0}$, with values of $55 \pm 10$ from type Ia supernovae being reported by Gustav Tammann, and $73 \pm 6$ (statistical) \pm 8 (systematic) km/sec/Mpc by our group (and based on a number of independent secondary methods). 
Perhaps most importantly, the error bars now overlap and all groups are now quoting both statistical as well as systematic errors.

Our systematic error takes into account a number of factors including: the present uncertainty in the zero point of the Cepheid period-luminosity relation of $\pm 5 \%$ (effectively the uncertainty in the distance to the LMC), the uncertainty due to metallicity in the Cepheid period-luminosity relation at a level of $\pm 5 \%$, an uncertainty of $\pm 7 \%$ that allows for the possibility that the locally measured $\mathrm{H}_{0}$ out to $\sim 10,000 \mathrm{~km} / \mathrm{sec}$ may not be the global value of $\mathrm{H}_{0}$, plus an allowance for a scale error in the photometry that could affect all of the results at the level of $\pm 3 \%$. At the present time, the total uncertainties amount to about $\pm 15 \%$. This result is based on a variety of methods, including a Cepheid calibration of the Tully-Fisher relation, type Ia supernovae, a calibration of distant clusters tied to Fornax, and direct Cepheid distances out to $\sim 20 \mathrm{Mpc}$.

In the next couple of years, all of the observations and analysis of $\mathrm{H}_{0}$ Key Project galaxy sample will have been undertaken, and a final calibration of secondary distance indicators can be completed. In addition, new, near-infrared H-band $(1.6 \mu \mathrm{m})$ NICMOS observations are now being scheduled on HST that will minimize the dominant sources of systematic uncertainty in the Cepheid distances (currently reddening and metallicity). New, optical and infrared photometry are being obtained for the Cepheid TullyFisher calibrators. New data are being obtained for the distant Tully-Fisher galaxies. Ground-based studies are dramatically increasing the numbers of well-observed supernovae. There are now quantitative reasons for optimism that the extragalactic distance scale will soon be firmly established at the $\pm 10 \%$ level.

\section{Acknowledgments}

We are pleased to acknowledge the enormous efforts of our co-investigators on the $\mathrm{H}_{0}$ Key Project team: L. Ferrarese, H. Ford, J. Graham, M. Han, J. Hoessel, J. Huchra, S. Hughes, G. Illingworth, R. Phelps, A. Saha, S. Sakai, N. Silbermann, and P. Stetson, and graduate students F. Bresolin, P. Harding, D. Kelson, L. Macri, D. Rawson, and A. Turner. This work is based on observations with the NASA/ESA Hubble Space Telescope, obtained by the Space Telescope Science Institute, which is operated by AURA, Inc. under NASA contract No. 5-26555. Support for this work was provided by NASA through grant GO-2227-87A from STScI. 


\section{References}

Aaronson, M. (1983) in Highlights of Astronomy as Presented at the XVIIIth General Assembly of the IAU, ed. West, R.M., 6, p. 269

Alcock, C. et al. (1997), Astrophys. J., 482, 89-97

Baum, W.A., Hammergren, M., Thomsen, B., Groth, E.J., Faber, S.M., Grillmair, C.J. \& Ajhar, E.A. (1997), Astron. J., 113, pp. 1483-1494

Beaulieu, J.P., Krockenberger, M., Sasselov, D.D., Renault, C., Ferlet, R., Vidal-Madjar, A., Maurice, E., Prevot, L., et al. (1997), Astron. Astrophy., 321, pp. L5-L8

Caldwell, J.A.R. \& Laney, C.D. (1991), IAU Symposium No. 148: The Magellanic Clouds, ed. R. Haynes and D. Milne (Dordrecht: Kluwer) pp. 249-257

Chiosi, C., Wood, P., \& Capitanio, N. (1993) Astrophys. J. Suppl., 86, p. 541

Donahue, M. \& Livio, M. (eds.) (1997) The Extragalactic Distance Scale. Cambridge University Press, Cambridge

Feast, M.W. \& Catchpole, R.M. (1997) Mon. Not. Royal Astr. Soc., 286, pp. L1-L5

Feldmeirer, J.J., Ciardullo, R. \& Jacoby, G. H. (1997), Astrophy. J., 479, pp. 231-243

Ferrarese, L., Freedman, W.L., Hill, R.J., Saha, A., Madore, B.F., Kennicutt, Jr., R.C., Stetson, P.B., Ford, H.C., et al. (1996), Astrophys. J., 464, pp. 568-599

Freedman, W. L. (1990), Astrophy. J., 355, pp. L35-L38

Freedman, W.L. \& Madore, B.F. (1990) Astrophys. J., 365, p. 186

Freedman, W.L. \& Madore, B.F. (1993), in New Perspectives on Stellar Pulsation and Pulsating Variable Stars, eds. Nemec, J.M. \& Matthews, J.M. IAU Colloquium 139, Cambridge University Press, Cambridge, pp. 61-71

Freedman, W.L. \& Madore, B.F. (1996), in Clusters, Lensing, and the Future of the Universe. eds. Trimble, V. \& Reisenegger, A., ASP Conf. Ser., 88, pp. 9-28

Freedman, W.L., Madore, B.F., \& Kennicutt, R.C. (1997) in The Extragalactic Distance Scale, eds. Donahue, M. \& Livio, M. Cambridge University Press, Cambridge, pp. 171-185

Freedman, W.L., Madore, B.F., Mould, J.R., Hill, R., Ferrarese, L., Kennicutt Jr., R.C., Saha, A., Stetson, P.B., Graham, J.A., et al. (1994a), Nature, 371, pp. 757-762

Freedman, W.L., Hughes, S.M., Madore, B.F., Mould, J.R., Lee, M.G., Stetson, P., Kennicutt, R.C., Turner, A., Ferrarese, L., Ford, H., et al. (1994b), Astrophys. J., 427, pp. 628-655

Sekiguchi, M. \& Fukugita, M. (1997) Observatory, submitted.

Giovanelli. et al. (1997) Astrophys. J. Lett., 477, pp. L1-L4

Gould, A. (1994) Astrophys. J., 426, p. 542

Gould, A. \& Uza, O. 1997, Astrophys. J., submitted (=astro-ph/9705051)

Graham,J. et al. (1997), Astrophys. J., 477, pp. 535-559

Hamuy, M., Phillips, M.M., Maza, J., Suntzeff, N.B., Schommer, R.A., \& Aviles, R. (1995), Astrophys. J., 109, pp. 1-13

Huchra, J. (1988) in Extragalactic Distance Scale, eds van den Bergh, S. \& Pritchet, C.J. Pub. Astron. Soc. Pacif. Conf. Series, 4, pp. 257-280

Hughes, S. M. et al. (1998) Astrophys. J., submitted.

Iben, I., Jr. \& Tuggle, R.S. (1975), Astrophy. J., 197, pp. 39-54 Properties of Cepheids in the Galaxy, in Andromeda, and in the Magellanic Clouds

Jerjen, H., \& Tammann, G.A. (1993), Astron. Ap., 276, pp. 1-8

Jacoby, G. et al. (1992) Pub. Astron. Soc. Pacif., 104, p. 599

Kelson, D.D., Illingworth, G.D., Freedman, W.F., Graham, J.A., Hill, R., Madore, B.F., Saha, A., Stetson, P.B., et al. (1996), Astrophys. J., 463, pp. 29-59

Kelson, D.D., Illingworth, G.D., Freedman, W.F., Graham, J.A., Hill, R., Madore, B.F., Saha, A., Stetson, P.B., et al. (1997), Astrophys. J., 478, 430

Kennicutt, R. C., Freedman, W. L., \& Mould, J. R. (1995), Astron. J. 110, pp. 1476-1491

Kennicutt, R. C., et al. (1998) Astrophys. J., in press, (astro/ph 9712055)

Jacoby, G. et al. (1992) Pub. Astron. Soc. Pacif., 104, p. 599

Kochanek, C. S. (1997) Astrophys. J., in press 
Madore, B.F. (1983) in Highlights of Astronomy as Presented at the XVIIIth General Assembly of the IAU, ed. R.M. West, 6, pp. 217

Madore, B. F.\& Freedman, W. L. (1991) Pub. Astron. Soc. Pacif., 103, pp. 933-957

Madore, B. F.\& Freedman, W. L. (1997) Astrophys. J., Jan. 1, submitted.

Madore, B.F., Freedman W.L., \& Sakai, S. (1997) in The Extragalactic Distance Scales, eds. Donahue, M. \& Livio, M. Cambridge University Press, Cambridge.

Madore, B. F. et al. (1998) Nature, in preparation

Mould, J.R. et al. (1997) in The Extragalactic Distance Scale, eds. Donahue, M. \& Livio, M. Cambridge University Press, Cambridge

Mould, J., Huchra, J.P., Bresolin, F., Ferrarese, L., Ford, H.C., Freedman, W.L., Graham, J., Harding, P., Hill, R., et al. (1995), Astrophys. J., 449, pp. 413-421

Newman, J., Zepf, S., Freedman W.L., Madore, B.F., Davis, M. \& Phelps, R. (1998) Astrophys. J., in preparation.

Panagia, N., et al. (1996), in The Extragalactic Distance Scale, poster papers from the STScI Symposium, eds. M. Livio \& M. Donahue.

Phelps, R. et al. (1998), Astrophys. J., in press.

Phillips, M. (1993) Ap.J., 413, L105-L108

Rawson, D. et al. (1997), Astrophys. J., 490, pp. 517-556

Reiss, A., Press, W. \& Kirshner, R. (1995) Ap.J., 438, L17-L20

Saha, A., Sandage, A., Labhardt, L., Tammann, G.A., Macchetto, F.D., \& Panagia, N. (1994), Astrophys. J., 425, pp. 14-34

Saha, A., Sandage, A., Labhardt, L., Schwengeler, H., Tammann, G.A., Panagia, N., \& Macchetto, F.D. (1995), Astrophys. J., 438, pp. 8-26

Saha, A., Sandage, A., Labhardt, L., Tammann, G.A., Macchetto, F.D., \& Panagia, N. 1996, Astrophys. J., 466, pp. 55-91

Sakai, S., Madore, B.F. \& Freedman, W. L. (1996), Astrophy. J., 461, pp. 713-723

Sandage, A., Saha, A., Tammann, G.A., Labhardt, L., Panagia, N., \& Macchetto, F.D. (1996), Astrophys. J. Lett., 460, pp. L15-L18

Sasselov, D.D., Beaulieu, J.P., Renault, C., Grison, P., Ferlet, R., Vidal-Madjar, A., Maurice, E., Prevot, L., et al. (1997), Astron. Astrophy., 324, pp. 471-482

Schechter, P.L., Mateo, M., \& Saha, A. (1993) Publ. Astr. Soc. Pac., 105, 1342

Stetson, P.B. (1994) Astron. J., 106, 205

Silbermann, N. et al. (1996), Astrophys. J., 470, pp. 1-37

Silbermann, N. et al. (1998), Astrophys. J., in preparation

Stothers, R. (1988) Astrophys. J., 329, p. 712

Tammann, G.A. \& Sandage, A. (1983), in Highlights of Astronomy as Presented at the XVIIIth General Assembly of the IAU, ed. West, R.M. 6, pp. 301

Tanvir, N.R., Shanks, T., Ferguson, H.C., \& Robinson, D.R.T. (1995), Nature, 377, pp. 27-31

Tonry, J.L., Blakeslee, J.P., Ajhar, E.A., \& Dressler, A. (1997), Astrophys. J, 475, pp. 399-413

Trimble, V. (1987) Astron. Rev. Astrophys., 25, p. 425

Westerlund, B.E. (1997) The Magellanic Clouds, Cambridge Astrophysics Series: 29. Cambridge University Press, Cambridge 